\title{
Characterization of the ocular surface
} temperature dynamics in glaucoma subjects
using long-wave infrared thermal imaging

\author{
Nery García-Porta, ${ }^{1, *}$ Francisco Javier Gantes-Nuñez, ${ }^{1,2}$ Juan Tabernero, ${ }^{1,3}$ and Shahina Pardhan ${ }^{1}$ \\ ${ }^{1}$ Vision and Eye Research Institute (VERI) - School of Medicine, Anglia Ruskin University, CB1 2LZ Cambridge, UK \\ ${ }^{2}$ Centro de Investigaciones en Óptica, C.P. 37150, León, Gto., Mexico \\ ${ }^{3}$ Departamento de Electromagnetismo y Electrónica, Universidad de Murcia, 30100 Murcia, Spain \\ *Corresponding author: nery.garcia-porta@anglia.ac.uk
}

Received 15 November 2018; revised 18 April 2019; accepted 23 April 2019; posted 24 April 2019 (Doc. ID 352043); published 17 May 2019

\begin{abstract}
We evaluated the dynamics of ocular surface temperature using thermal imaging in $\mathbf{2 1}$ glaucoma subjects and 19 healthy subjects. On opening of the eye, subjects with glaucoma showed significantly cooler temperatures in the central cornea compared to the control group. The upper eyelid was also significantly cooler just before the eye opened. Immediately after opening the eye, the dynamic of temperature change was different in the two groups. In subjects with glaucoma, the eyes cooled significantly faster, with an average decrease of $0.49^{\circ} \mathrm{C}$ during the first second compared to $0.24^{\circ} \mathrm{C}$ in the control group. Our results support the hypothesis that both the stability of the tear film and changes in the ocular blood supply in subjects with glaucoma play an important role in thermal dynamics of the ocular surface. () 2019 Optical Society of America
\end{abstract}

\section{INTRODUCTION}

Glaucoma is an optic neuropathy characterized by the progressive degeneration of the optic nerve. Approximately 64.3 million people worldwide have glaucoma, and this number is predicted to escalate to 111.8 million in 2040 due to the aging population [1]. Glaucoma is a painless disease in general, but without treatment it can lead to substantial visual field loss and blindness [2].

The pathogenesis of the disease is commonly associated with a "vascular" theory, suggesting that glaucoma is due to an impaired blood supply in the eye. The optic nerve damage is assumed to be the consequence of microvascular injuries and low ocular perfusion pressure [the difference between intraocular pressure (IOP) and systemic blood pressure] [3,4].

Treatments for glaucoma are mostly focused on lowering IOP based on the regular use of topical eye drops [5-7]. As glaucoma is a progressive disease, long-term treatment is required for its control. Although there are a great variety of hypotensive eye drops in the market, frequent side effects affecting the ocular surface are reported, mostly as dry eye, occurring in up to $59 \%$ of subjects [8-10].

Dry eye disease (DED) is a multifactorial disease that presents with tear film instability and ocular dryness symptoms [11]. The tear film provides the cornea with nutrients, and protects and moisturizes the ocular surface. The systematic use of glaucoma eye drops may alter the quality and functionality of this ocular layer [8]. An unstable tear film can decrease the quality of vision, which in turn affects daily activities like reading or driving as well as producing symptoms of dryness and discomfort $[10,12,13]$.

The most commonly used method to assess tear stability is FBUT (fluorescein tear break-up time), which requires the instillation of fluorescein onto the tear film. However, the amount of fluorescein used in the test may alter the results obtained. Also, this test requires a subjective assessment of the tear stability [14].

A non-invasive test that can give objective results is based on long-wave infrared thermal imaging of the ocular surface. This is a promising technology with the potential to improve the diagnosis and follow-up of several eye pathologies. For instance, ocular thermography is able to detect inflammatory eye diseases that typically present an elevated ocular surface temperature (OST) [15]. Galassi et al. found lower OST in glaucoma subjects compared to healthy subjects, and the authors suggested that this could be related to hemodynamic alterations in the optic nerve [16]. Other studies have also shown that the OST is lower and the cooling rate faster in DED subjects than in healthy subjects [17-19]. As DED is reported in subjects with glaucoma, it is possible that the low OST reported by Galassi is due to the eye drops. Therefore, a better understanding of the continuous dynamical changes in the OST might help to classify the differential features of the ocular surface in glaucoma. These features could be related to changes in the tear film dynamics, both in the center of the cornea (where there is no vascularization) and in peripheral vascular zones (conjunctiva and limbus areas). 
The purpose of this study is to assess the OST in different areas of the ocular surface (vascular and avascular zones), and to examine the continuous dynamical changes in glaucoma and healthy subjects.

\section{METHODS}

The prospective study was conducted in agreement with the tenets of the Declaration of Helsinki, and the study protocol was reviewed and approved by the Ethics Committee of Medical Science Faculty of Anglia Ruskin University (ARU). Following an explanation of the nature and possible consequences of the study, all participants signed a consent form before being enrolled in the study.

To avoid fluctuations of the environmental conditions, all tests were performed in a controlled environmental chamber (CEC) (PSR-B, WEISS Gallenkamp) based in the Vision and Eye Research Institute at Anglia Ruskin University. The CEC has the capacity to maintain a set temperature between $5^{\circ} \mathrm{C}$ and $40^{\circ} \mathrm{C}\left( \pm 1^{\circ} \mathrm{C}\right.$ from set value $)$ and a relative humidity between $5 \%$ and $85 \%$ ( $\pm 5 \%$ from set value). The air in the CEC circulates constantly, with a laminar flow of $0.08 \mathrm{~m} / \mathrm{s}$. The measurements were performed at $45 \%$ of relative humidity and $23^{\circ} \mathrm{C}$. Before doing the tests, the participants sat in the CEC for a $10 \mathrm{~min}$ equilibration period so that the body could adapt to the room temperature.

\section{A. Subjects}

The glaucoma group was recruited from the Vision and Eye Research Institute (VERI) clinics at ARU and from the International Glaucoma Association. The participants in the control group were recruited from VERI clinics and members of ARU and University of Cambridge. Twenty-one glaucoma subjects using glaucoma eye drops for at least six months prior to data collection and 19 age-matched control subjects without any eye disease were recruited. Exclusion criteria included any ocular surgery in the last two years, history of corneal refractive surgery, any acute or chronic ocular disease (except glaucoma), eye allergy, use of any topical medications apart from glaucoma eye drops or artificial tears, systemic diseases (e.g., diabetes) or any systemic medications (e.g., anxiolytics) that may produce DED.

\section{B. Thermography}

Thermal videos were acquired using a long wave $(17 \mu \mathrm{m})$ infrared thermal camera (Therm-App Hz, Opgal Optronic Industries Ltd., Israel). The frame rate was $25 \mathrm{~Hz}$, with a spatial resolution of $384 \times 288$ pixels and high thermal sensitivity $\left(<0.07^{\circ} \mathrm{C}\right)$. Video recordings were taken for $8 \mathrm{~s}$ once the subjects opened their eyes after having kept them shut for around $10 \mathrm{~s}$. After opening the eyes, the participants were requested to blink normally while their head was resting on a chinrest and they were looking at a fixed point straight ahead. Examples of the images collected are shown in Fig. 1. The thermal images were analyzed frame by frame using MATLAB. Three different parameters were evaluated.

(1) The baseline temperature (T0) taken immediately after opening the eye $(t=0 \mathrm{~s})$. The average temperature was calculated over two ocular surface zones, central cornea and the periphery of the ocular surface that excluded the central cornea (this area was manually marked with the maximum ellipse that covered the ocular surface). In addition, the temperature of the central superior eyelid just before the eye opened was measured. These three zones are shown in Fig. 2. In all three cases the diameter of the circular area (included or excluded) was $3 \mathrm{~mm}$.

(2) Changes in temperature over time in the central and peripheral ocular surface (Fig. 2). The temperatures were evaluated at $0,0.5,1,1.5,4$, and $8 \mathrm{~s}$ after opening the eye.

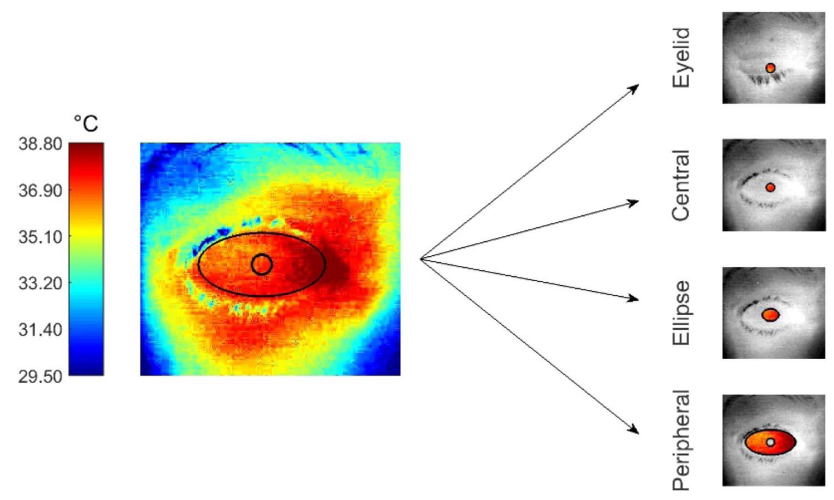

Fig. 2. Areas where the temperature was evaluated.

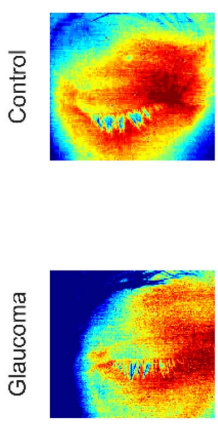

$\mathrm{t}<0$ s
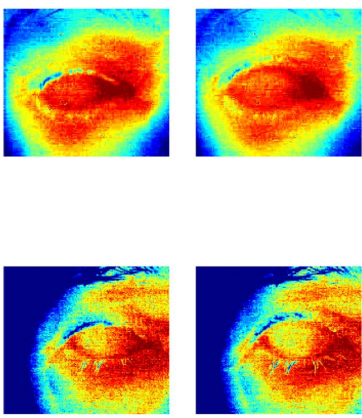

$\mathrm{t}=0 \mathrm{~s}$

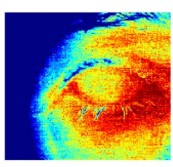

$t=1$ s
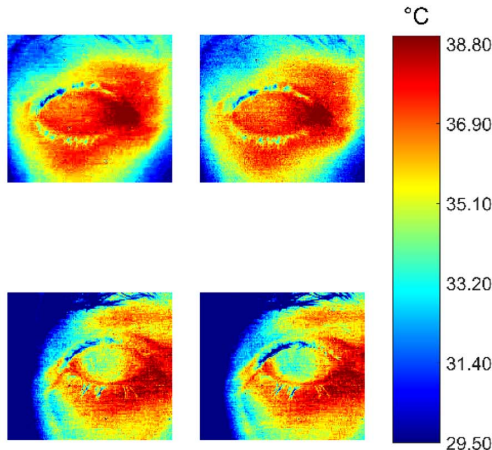

$\mathrm{t}=4 \mathrm{~s}$

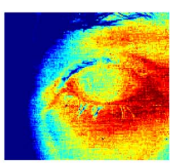

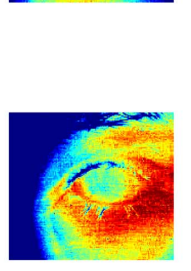

$t=8 s$

Fig. 1. Example of thermal images collected in an eye with glaucoma and in a healthy eye before opening the eye, and then after 1,4 , and $8 \mathrm{~s}$ after opening the eye. Thermal scale runs from $29.5^{\circ} \mathrm{C}$ (blue tones) to $38.8^{\circ} \mathrm{C}$ (red tones). 
(3) The cooling rate in the central cornea was calculated as the slope of the linear function at three different time intervals after opening the eye: (i) from 0 to $2 \mathrm{~s}$, (ii) from 2 to $4 \mathrm{~s}$, and (iii) from 4 to $8 \mathrm{~s}$. As the participants could blink normally after opening the eye, all the frames that corresponded to a blink after opening the eye were manually excluded from this analysis. The cooling rate was analyzed in an elliptical area (major axis: $5 \mathrm{~mm}$; minor axis: $3 \mathrm{~mm}$ ) located in the center of the cornea (Fig. 2).

\section{DED Clinical Tests}

In addition, two commonly used clinical tests were performed: FBUT to assess tear stability and Schirmer test to measure the tear production.

FBUT was measured by instilling fluorescein into the lower fornix (Bio Fluoro Fluorescein Sodium Ophthalmic Strips, Biotec Vision Care). To measure the FBUT, a slit lamp (Symphony, Keeler Optics Ltd.) was used with a yellow filter to enhance the contrast. One minute after instilling the fluorescein, the participants were asked to blink a couple of times, and then were requested to keep the eyes open for as long as possible. The procedure was repeated three times to obtain a more reliable value.

Schirmer test was performed without anesthesia. A sterile Schirmer strip (I-DEW Tearstrips, Entod Research Cell UK, Ltd.) was placed in the temporal tarsal conjunctiva of the lower eyelid, and the participants were requested to keep their eyes closed for $5 \mathrm{~min}$. The length of the wet strip area was measured.

\section{Statistics}

Statistical analysis was conducted using SPSS (SPSS Inc., Chicago, Illinois, USA). Shapiro-Wilk test was used to evaluate the normal data distribution. Independent sample $\mathrm{T}$ test or Mann-Whitney $U$ test, depending on the data distribution, were used to assess differences between both groups for T0, FBUT, and Schirmer. Mixed ANOVA with one betweenvariable (group) and one within-variable (time) was used to evaluate the changes in cooling rate. Mixed ANOVA with one between-variable (group) and two within-variables (time and zone) was used to evaluate the temperature changes in the central cornea and in the periphery of the ocular surface. When statistically significant differences were found, Bonferroni posthoc correction was applied. The correlations were analyzed using the Pearson test.

To avoid data repetition and reduce the time of the experiment, data were collected only in the right eye (except if the glaucoma participant was using hypotensive eye drops in the left eye only, in which case the left eye was examined) of each participant. $P$ values lower than 0.05 were considered statistically significant.

\section{RESULTS}

Figure 3 shows the temperatures taken from the superior eyelid [Fig. 3(a)] just before the eye opened, the central corneal area [Fig. 3(b)], and the periphery of the ocular surface [Fig. 3(c)] immediately after opening the eye. Eyes with glaucoma were significantly cooler in the superior eyelid $(P=0.046)$ and central cornea $(P=0.036)$. The difference in the peripheral ocular surface between both groups was not significant $(P=0.183)$.

Figure 4 shows the correlation between the temperatures in different parts of the ocular surface. The baseline temperature (T0) in the center of the cornea was significantly correlated with that from the eyelid and the peripheral ocular surface $(P<0.001)$, and T0 in the periphery was significantly correlated with T0 in the eyelid as well $(P<0.001)$.

Once the eye was open, the decrease in central cornea temperature was greater in glaucoma than in healthy subjects $(F=12.322, P=0.001)$. The average decrease was $0.49^{\circ} \mathrm{C}$ in glaucoma and $0.24^{\circ} \mathrm{C}$ in healthy subjects during the first second after opening the eye. A slower decrease was then observed between 1 and 8 seconds [Fig. 5(a)]. This cooling behavior was analyzed in more detail by calculating the cooling rate at three different time intervals: from 0 to $2 \mathrm{~s}, 2$ to $4 \mathrm{~s}$, and 4 to $8 \mathrm{~s}$ [Figs. 5(b), 5(c), and 5(d)]. The average cooling rate during the

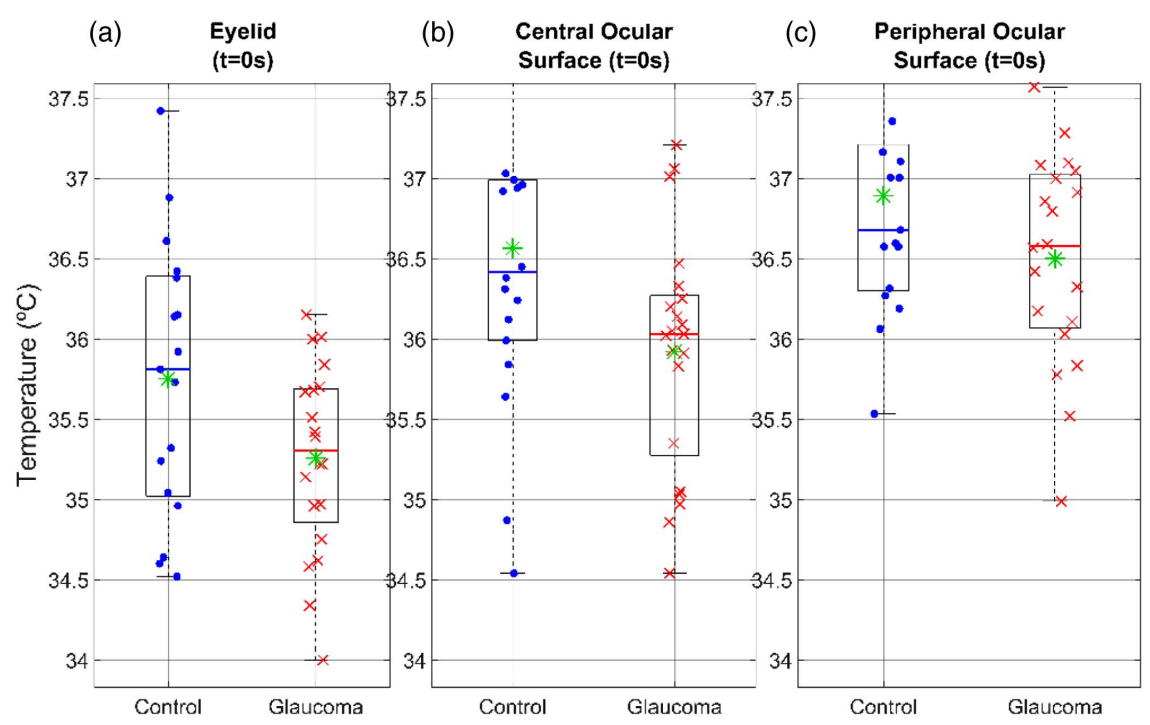

Fig. 3. Values of the temperature measured in both groups, control and glaucoma, in different areas of the eye. (a) Superior eyelid just before opening the eye; (b) center of the cornea and (c) periphery of the ocular surface just after opening the eye. Data are shown as circles and crosses for healthy and glaucomatous eyes, respectively. 
(a)

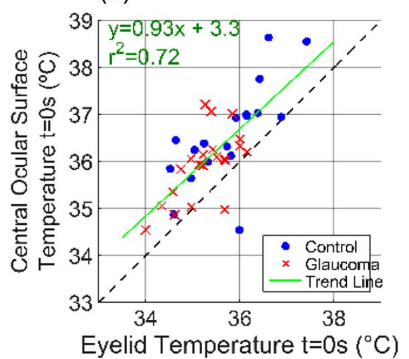

(b)

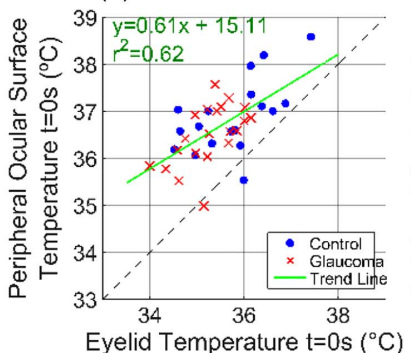

(c)

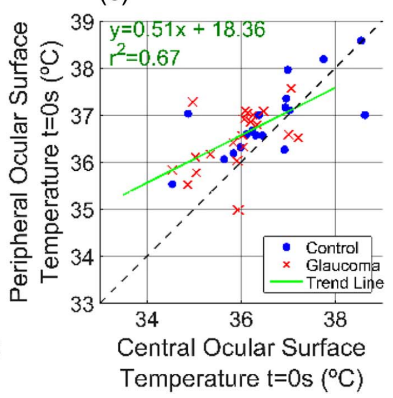

Fig. 4. Correlation between temperature in (a) the superior eyelid and center of the cornea, (b) the superior eyelid and periphery of the ocular surface, and (c) center of the cornea and the periphery of the ocular surface.
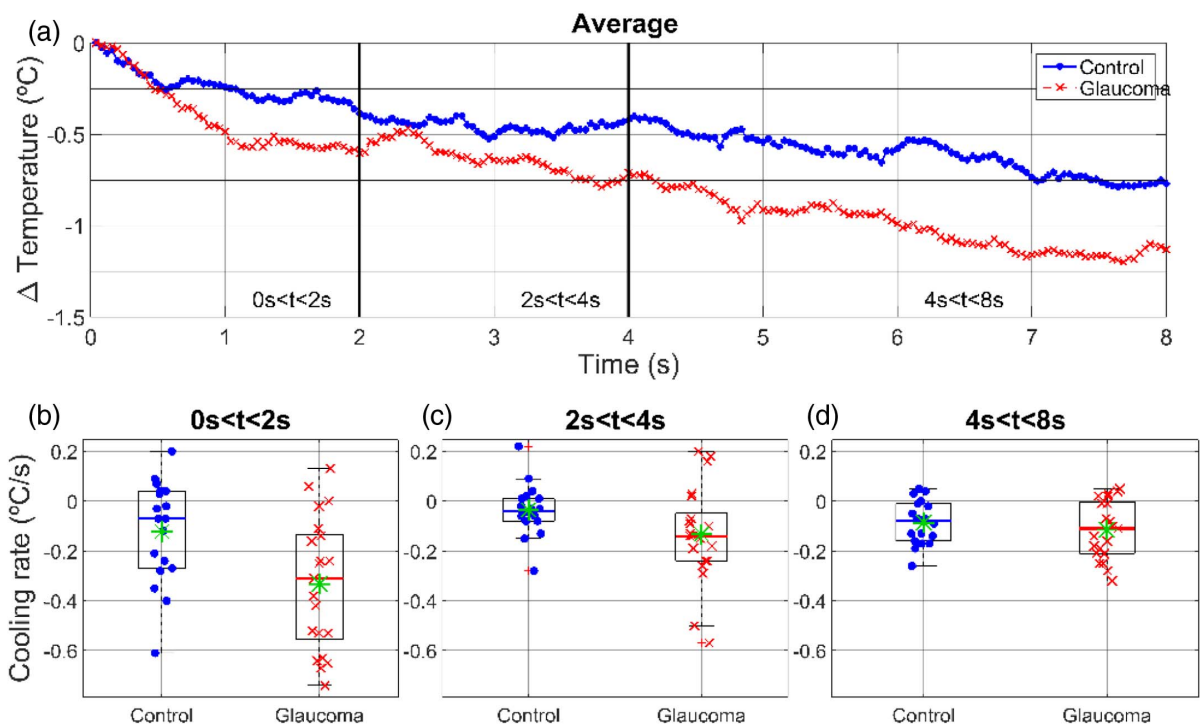

Fig. 5. (a) Average ocular surface temperature changes in the central cornea in both groups, glaucoma and control. Cooling rate (b) during the first two seconds, (c) between seconds 2 and 4, and (d) between seconds 4 and 8 after opening the eye.

first two seconds was -0.333 and $-0.122^{\circ} \mathrm{C} / \mathrm{s}$ in the glaucoma and control groups, respectively. For the interval between the second and fourth seconds, it was -0.133 and $-0.035^{\circ} \mathrm{C} / \mathrm{s}$, and for the interval between the fourth and eighth seconds the rate was -0.115 and $-0.085^{\circ} \mathrm{C} / \mathrm{s}$ in glaucoma and healthy subjects, respectively. Statistical differences existed between these intervals $(F=7.549, P=0.001)$, with a faster cooling rate during the first two seconds. The interaction between time interval and group was not significant (Table 1).

Figure 6 shows the decrease of temperature in the central cornea (panel a) and the peripheral ocular surface (panel b) for T0 (immediately after opening the eye) and at $0.5,1$, $1.5,4$, and $8 \mathrm{~s}$ after opening the eye. The rate of change of temperature was significantly greater in the glaucoma group compared to the control group for both central and peripheral ocular surface $(F=13.978, P=0.001)$. On average, eight seconds after opening the eye, the drop of temperature in the central cornea was $-1.11^{\circ} \mathrm{C}$ in glaucoma and $-0.64^{\circ} \mathrm{C}$ in healthy subjects. In the peripheral ocular surface, the drop of temperature was $-1.15^{\circ} \mathrm{C}$ and $-0.55^{\circ} \mathrm{C}$ in glaucoma and control group, respectively. The temperature in the periphery was always higher than the temperature in the center in both groups (panels c and $\mathrm{d}$ ).

On comparing the clinical tests for DED, although the values obtained in this study are quite low in both groups, the glaucoma group shows significant FBUT values and nearly significant lower Schirmer values (Table 2).

\section{Table 1. Results of the Repeated Measures ANOVA}

\section{Cooling Rate $\left({ }^{\circ} \mathrm{C} / \mathrm{s}\right)$ in the Central Cornea $(1.5 * 2.5 \mathrm{~mm})$}

\begin{tabular}{lc}
\hline Group (control, glaucoma) & $F=12.322, P=0.001$ \\
Time (0-2 s, 2-4 s, 4-8 s) & $F=7.549, P=0.001$ \\
Time* group & $F=2.529, P=0.087$ \\
\hline$\Delta$ Temperature in two different areas: central cornea and \\
peripheral ocular surface & $F=13.978, P=0.001$ \\
\hline Group (control, glaucoma) & $F=55.715, P<0.001$ \\
Time (0 s, 0.5 s, 1 s, 1.5 s, 4 s, 8s) & $F=12.391, P=0.001$ \\
Area (center, periphery) & $F=2.028, P=0.163$ \\
Area*Group & $F=1.280, P=0.274$ \\
Time*area & $F=6.071, P<0.001$ \\
Group*time & \\
\hline
\end{tabular}



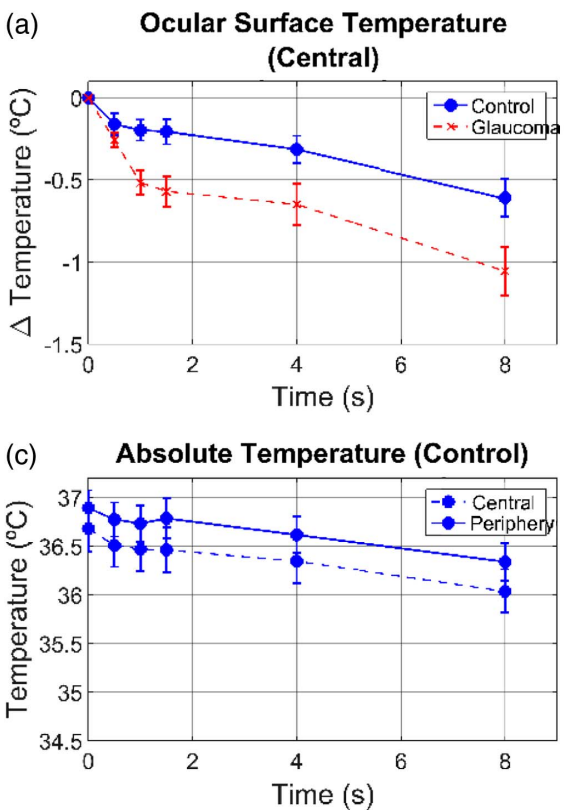

(b) Ocular Surface Temperature (Periphery)

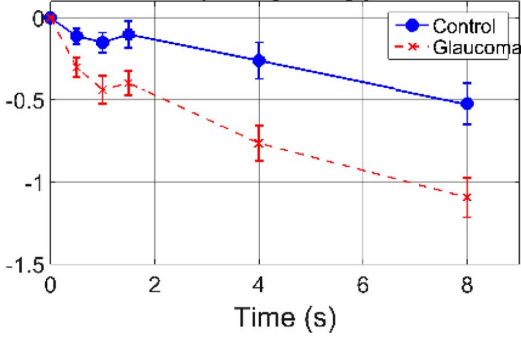

(d) Absolute Temperature (Glaucoma)

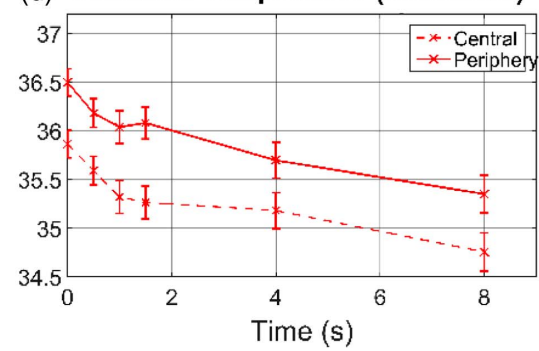

Fig. 6. Temperature changes $0.5,1,4$, and $8 \mathrm{~s}$ after opening the eye in (a) central cornea and (b) periphery of the ocular surface.

Table 2. Values of Schirmer and FBUT Tests in Glaucoma and Healthy Subjects

\begin{tabular}{lccc}
\hline & Control Group (mean $\pm \mathbf{s d})$ & Glaucoma Group (Mean $\pm \mathbf{s d}$ ) & Statistical Differences between Groups \\
\hline FBUT $(s)$ & $6.053 \pm 2.223$ & $4.476 \pm 2.159$ & $t=2.274, P=0.029$ \\
Schirmer $(\mathrm{mm})$ & $9.176 \pm 9.382$ & $4.737 \pm 4.805$ & $U=101.000, P=0.052$ \\
\hline
\end{tabular}

\section{DISCUSSION}

\section{A. Glaucoma Eyes Are Colder than Normal Eyes}

On average, the central corneas of glaucomatous eyes were $0.64^{\circ} \mathrm{C}$ cooler than in healthy eyes. This is in agreement with a previous study by Galassi et al., who also found lower temperatures $\left(0.57^{\circ} \mathrm{C}\right)$ in the central cornea of the glaucomatous eyes compared to controls [16]. In our present study, we found that the temperature of the upper eyelid was also lower in subjects with glaucoma. There are various reasons to explain these data. First, it could be that glaucomatous eyes have an impaired blood supply not only to the optic nerve but also to the entire eye, including the vascular systems that supply blood to the choroidal, conjunctiva, orbit, lids, and limbus [3]. While the central cornea does not have a vascular system, its temperature is strongly correlated to that of the eyelid as shown in Fig. 4.

The second possible explanation could be related to the glaucoma medication. It is well known that different hypotensive drops present diverse local and systemic side effects, and some of them affect blood pressure [7], which in turn could influence the ocular surface temperature [20]. A decrease in the temperature of the ocular surface may lead to a decrease in the quality of the tear film since secretions from the glands such as the meibonium glands are negatively affected by lower temperatures [21]. The participants in this study used a wide variety of glaucoma medications. The relatively small size of our sample precluded any further analysis on effects of medication, as different drugs may have different side effects.

The third possibility is that cooler temperatures in glaucomatous eyes were due to intrinsic DED in these subjects in addition to glaucoma. Certainly, our glaucoma subjects showed more signs of DED, as suggested by a shorter BUT and lower Schirmer values than the healthy subjects. On average, the literature shows that subjects with DED have temperatures of around one degree lower than healthy subjects [17]. Glaucoma subjects in our study do show signs of DED, but whether this is caused by hypotensive eye drops or is due to the fact that they have glaucoma cannot be ascertained directly from the study. However, the most likely explanation is that a combination of all these three factors (glaucoma, side effects of the glaucoma eye drops, and DED) caused the overall cooler temperatures in subjects with glaucoma.

\section{B. Differences in the Ocular Surface Temperature Dynamics of Glaucoma and Normal Eyes}

Cooling rate of the central cornea was calculated at three different temporal intervals. First, a sudden decrease of temperature occurred immediately after opening the eye. Craig et al. attributed a rapid decrease in corneal temperature to a positive latent heat of vaporization of the tear film and consequently to a higher evaporation rate [22]. This initial and rapid cooling rate was greater in glaucoma than in healthy subjects. However, a bilinear tendency was observed [Fig. 5(a)] in both groups, showing the rapid initial decrease and a more attenuated cooling phase. The transition 
point between both phases occurred later (about $1 \mathrm{~s}$ after opening the eye) in glaucoma subjects compared to healthy subjects, whose initial cooling phase last only about $0.5 \mathrm{~s}$ on average.

During the second cooling phase, the temperature continued to drop although with a slower rate in both groups. It is likely that the lower ambient temperature of the environment $\left(23^{\circ} \mathrm{C}\right)$ with respect to the temperature in the corneal surface was responsible for this decrease in temperature [23].

Significant differences between glaucoma and control subjects were found in the dynamic of change of central cornea temperature. Considering that the central cornea does not have a vascular system, after opening the eye, the changes in the temperature of the central cornea were most likely influenced by ambient environmental temperature and tear film characteristics. Since the ambient temperature was the same for all subjects (in the controlled environmental chamber), it is likely the faster cooling rate found in glaucoma suggests some instability of the tear film [24]. In fact, glaucoma group did show shorter FBUT values. In this regard, glaucoma subjects show similar behavior to those with DED [17-19]. Further studies that would analyze the thermal characteristics of the ocular surface before and after commencement of glaucoma eye drops are needed to assess if these thermal characteristics are due to the glaucoma itself or by the effects of topical glaucoma medication.

The analysis taken in the peripheral ocular surface area (excluding the central avascular zone) showed similar dynamics to the central cornea (Fig. 6). Again, the drop in the temperature here was higher in the glaucoma group than in the control group. The faster temperature decrease found in the periphery in glaucoma subjects suggests that this vascular zone is also affected. The baseline temperature in the center of the cornea showed a strong correlation with the baseline temperature of the eyelid and the peripheral ocular surface, suggesting that central corneal temperature is affected not only by the tear film, but also by the ocular vascular system.

\section{CONCLUSIONS}

The baseline temperature is cooler in glaucomatous eyes compared to healthy eyes. The cooling rate is also faster in glaucoma than in healthy subjects. In addition, the temperature of the peripheral area of the ocular surface which is vascular (conjunctiva and limbus) tended to change more in glaucoma than in healthy subjects (but this was not significant). In general, we hypothesize that, although the tear film characteristics have an important influence on the ocular surface temperature, alterations in the ocular blood supply in glaucoma subjects also play a significant role in the thermal dynamics of the ocular surface.

Funding. European Union's Horizon 2020 research and innovation programme under H2020 Marie SkłodowskaCurie Actions (747441).

Disclosures. The authors declare that there are no conflicts of interest related to this paper.

\section{REFERENCES}

1. Y. C. Tham, X. Li, T. Y. Wong, H. A. Quigley, T. Aung, and C. Y. Cheng, "Global prevalence of glaucoma and projections of glaucoma burden through 2040: a systematic review and meta-analysis," Ophthalmology 121, 2081-2090 (2014).

2. A. Tuulonen, P. J. Airaksinen, E. Erola, E. Forsman, K. Friberg, M. Kaila, A. Klemetti, M. Makela, P. Oskala, P. Puska, L. Suoranta, H. Teir, H. Uusitalo, E. Vainio-Jylha, and M. L. Vuori, "The Finnish evidencebased guideline for open-angle glaucoma," Acta Ophthalmol. Scand. 81, 3-18 (2003).

3. M. Yanagi, R. Kawasaki, J. J. Wang, T. Y. Wong, J. Crowston, and Y. Kiuchi, "Vascular risk factors in glaucoma: a review," Clin. Exp. Ophthalmol. 39, 252-258 (2011).

4. A. K. Deb, S. Kaliaperumal, V. A. Rao, and S. Sengupta, "Relationship between systemic hypertension, perfusion pressure and glaucoma: a comparative study in an adult Indian population," Indian J. Ophthalmol. 62, 917-922 (2014).

5. J. B. Jonas, T. Aung, R. R. Bourne, A. M. Bron, R. Ritch, and S. Panda-Jonas, "Glaucoma," Lancet 390, 2183-2193 (2017).

6. D. Sambhara and A. A. Aref, "Glaucoma management: relative value and place in therapy of available drug treatments," Ther. Adv. Chronic Dis. 5, 30-43 (2014).

7. P. Kalouda, C. Keskini, E. Anastasopoulos, and F. Topouzis, "Achievements and limits of current medical therapy of glaucoma," Dev. Ophthalmol. 59, 1-14 (2017).

8. F. Perez-Bartolome, J. M. Martinez-de-la-Casa, P. Arriola-Villalobos, C. Fernandez-Perez, V. Polo, and J. Garcia-Feijoo, "Ocular surface disease in patients under topical treatment for glaucoma," Eur. J. Ophthalmol. 27, 694-704 (2017).

9. C. Baudouin, J. P. Renard, J. P. Nordmann, P. Denis, Y. Lachkar, E. Sellem, J. F. Rouland, V. Jeanbat, and S. Bouee, "Prevalence and risk factors for ocular surface disease among patients treated over the long term for glaucoma or ocular hypertension," Eur. J. Ophthalmol. 23, 47-54 (2012).

10. C. E. Saade, H. B. Lari, T. L. Berezina, R. D. Fechtner, and A. S. Khouri, "Topical glaucoma therapy and ocular surface disease: a prospective, controlled cohort study," Can. J. Ophthalmol. 50, 132-136 (2015).

11. J. P. Craig, K. K. Nichols, E. K. Akpek, B. Caffery, H. S. Dua, C. K. Joo, Z. Liu, J. D. Nelson, J. J. Nichols, K. Tsubota, and F. Stapleton, "TFOS DEWS II definition and classification report," Ocular Surf. 15, 276-283 (2017).

12. S. Kastelan, M. Tomic, K. Metez Soldo, and J. Salopek-Rabatic, "How ocular surface disease impacts the glaucoma treatment outcome," BioMed Res. Int. 2013, 696328 (2013).

13. K. S. Na, K. Han, Y. G. Park, C. Na, and C. K. Joo, "Depression, stress, quality of life, and dry eye disease in Korean women: a population-based study," Cornea 34, 733-738 (2015).

14. J. S. Wolffsohn, R. Arita, R. Chalmers, A. Djalilian, M. Dogru, K. Dumbleton, P. K. Gupta, P. Karpecki, S. Lazreg, H. Pult, B. D. Sullivan, A. Tomlinson, L. Tong, E. Villani, K. C. Yoon, L. Jones, and J. P. Craig, "TFOS DEWS ॥ diagnostic methodology report," Ocular Surf. 15, 539-574 (2017).

15. A. A. Kawali, "Thermography in ocular inflammation," Indian J. Radiol. Imaging 23, 281-283 (2013).

16. F. Galassi, B. Giambene, A. Corvi, and G. Falaschi, "Evaluation of ocular surface temperature and retrobulbar haemodynamics by infrared thermography and colour Doppler imaging in patients with glaucoma," Br. J. Ophthalmol. 91, 878-881 (2007).

17. K. Abreau, C. Callan, R. Kottaiyan, A. Zhang, G. Yoon, J. V. Aquavella, J. Zavislan, and H. B. Hindman, "Temperatures of the ocular surface, lid, and periorbital regions of Sjogren's, evaporative, and aqueous-deficient dry eyes relative to normals," Ocular Surf. 14, 64-73 (2016).

18. T. Kamao, M. Yamaguchi, S. Kawasaki, S. Mizoue, A. Shiraishi, and Y. Ohashi, "Screening for dry eye with newly developed ocular surface thermographer," Am. J. Ophthalmol. 151, 782-791 (2011).

19. S. Matteoli, E. Favuzza, L. Mazzantini, P. Aragona, S. Cappelli, A. Corvi, and R. Mencucci, "Ocular surface temperature in patients with evaporative and aqueous-deficient dry eyes: a thermographic approach," Physiol. Meas. 38, 1503-1512 (2017).

20. X. Zhang, S. Vadoothker, W. M. Munir, and O. Saeedi, "Ocular surface disease and glaucoma medications: a clinical approach," Eye Contact Lens 45, 11-18 (2018).

21. W. H. Cho, I. C. Lai, P. C. Fang, C. C. Chien, S. L. Tseng, Y. H. Lai, Y. T. Huang, and M. T. Kuo, "Meibomian gland performance in 
glaucomatous patients with long-term instillation of IOP-lowering medications," J. Glaucoma 27, 176-183 (2018).

22. J. P. Craig, I. Singh, A. Tomlinson, P. B. Morgan, and N. Efron, "The role of tear physiology in ocular surface temperature," Eye (Lond.) 14, 635-641 (2000).
23. R. Mapstone, "Determinants of corneal temperature," $\mathrm{Br}$. J. Ophthalmol. 52, 729-741 (1968).

24. W. Li, A. D. Graham, S. Selvin, and M. C. Lin, "Ocular surface cooling corresponds to tear film thinning and breakup," Optom. Vision Sci. 92, e248-e256 (2015). 